\title{
Decoherence of a two-state atom driven by coherent light
}

\author{
Hyunchul Nha and H. J. Carmichael \\ Department of Physics, University of Auckland, Private Bag 92019, Auckland, New Zealand
}

\begin{abstract}
Recent studies of the decoherence induced by the quantum nature of the laser field driving a twostate atom [J. Gea-Banacloche, Phys. Rev. A 65, 022308 (2002); S. J. van Enk and H. J. Kimble, Quantum Inf. and Comp. 2, 1 (2002)] have been questioned by Itano [W. M. Itano, Phys. Rev. A 68, 046301 (2003)] and the proposal made that all decoherence is due to spontaneous emission. We analyze the problem within the formalism of cascaded open quantum systems. Our conclusions agree with the Itano proposal. We show that the decoherence, nevertheless, may be divided into two parts - that due to forwards scattering and to scattering out of the laser mode. Previous authors attribute the former to the quantum nature of the laser field.
\end{abstract}

PACS numbers: 03.67.Lx, 42.50.Ct

\section{INTRODUCTION}

Quantum computation, which relies on principles of quantum mechanics that find no analogue in a classical computer, has attracted much attention in recent years, and considerable effort has been devoted to implementing the elements of a quantum computer. Some quantum algorithms and quantum logic gates have been experimentally realized; for example, in an NMR system 1] and with trapped ions [2, 3, 4]. Trapped-ion systems, in particular, are considered promising, because of their scalability. The use of laser fields to manipulate two-state atomic transitions is crucial to these systems, since such manipulations realize the one- and two-qubit operations that form the basic building blocks of a quantum computation [5]. When modeling these manipulations, the laser fields are mostly taken to be classical; thus it is implicitly assumed that the interaction of the atom with the laser field contributes nothing to its decoherence. The same assumption, in fact, prevails beyond the quantum computation context, wherever the manipulation of an atomic state by a laser field is involved.

At a fundamental level, the laser should be treated as a quantum field, in which case its interaction with the atom would generally lead to entanglement of the two. It is natural then to ask whether the resolution of this entanglement (tracing out the laser field) leads to additional decoherence, i.e., in addition to the decoherence due to spontaneous emission. The issue can be framed from a quantum measurement point of view. One may measure the sum of the laser field and forwards-scattered field from the atom. This measurement would give, if not complete, then some information on the atomic state. The available information, whether the measurement is actually performed or not, would degrade the coherence of the atomic state, much as which-way information does in the Young's two-slit experiment. We are lead, therefore, to the question posed. The issue becomes particularly pressing when one considers a case where the atoms (ions) of a quantum computer are to be individually addressed by a tightly focused laser beam [3, 5]. The solid angle subtended by the laser field is then substantial, and a sig- nificant amount of information about the atomic qubits might be retrieved by measuring the forwards-scattered laser light.

The question raised has been asked by previous authors, with some disagreement in the answers $\underline{6}$, 7, 8, 9, 10. In this paper we address it in an alternative analysis, in a way that, for us, leads quickly to a clear conclusion, and helps resolve any remaining disagreement. We use the theory of cascaded open systems 11, 12, 13], first in its quantum trajectory formulation [12], and then as the starting point to derive a master equation for the driven atom. We show from the derived master equation that the rate of atomic decoherence is, indeed, given entirely by the rate of spontaneous emission for an atom driven by a classical field. We use quantum trajectories to expose the different scattering processes that contribute to the total rate: forwards scattering - scattering that overlaps the propagating laser pulse - and side scattering out of the laser pulse. Our treatment of the laser pulse is one dimensional, and therefore idealized. It nevertheless captures the essential character of the physics involvedthat the driving of an atom by a laser field is a scattering process. Decoherence accounts for the resolution of the Schrödinger entanglement between the scattering center and scattered fields. Assuming the driving field to be coherent, the latter, in simple language, is due to spontaneous emission. Our approach is applicable to nonclassical driving fields as well, where additional entanglement does arise.

We review the background in Sec. [II and formulate our treatment of the problem in Sec. III Analysis of the developed model is carried out in Secs. [V] and $\nabla$ Discussion of our results and their connection with earlier work is presented in Sec. VI

\section{BACKGROUND}

As mentioned, several authors have considered the entanglement of a coherently driven atom with its driving field [6, 7]. The previous works are carried out within the framework of the Jaynes-Cummings model. Their main result is that the entanglement is nonzero, although small 
for strong fields, scaling as $1 / \bar{n}$, where $\bar{n}$ is the mean number of photons in the laser pulse. The result has been questioned by Itano [8], who argues that there is no entanglement with the driving field at all. His argument is based on Mollow's treatment of resonance fluorescence 14], which, considering a quantized driving field in a coherent state, applies a displacement operator to the field to show that the atom equivalently couples to a classical driving field plus the quantized vacuum. Itano suggests that all entanglement originates in the interaction with the vacuum and is therefore resolved through spontaneous emission.

The first and single most important question arising from Itano's comment is whether or not the entanglement reported in [6, 7] results in decoherence that is additional to, or included within, the decoherence rate obtained from the standard treatment of spontaneous emission for an atom driven by a classical field. The assertion (implication) of Refs. [6, [7] is that it is additional to; Gea-Banacloche states so explicitly [9]: “... I wanted to focus, instead, on the decoherence due to the quantum nature of the laser field, which I took to be a separate source of error." Itano's position is that there is no decoherence in addition to spontaneous emission.

There is a second, more subtle question, featured most clearly in the reply of van Enk and Kimble [10]. Does the driven atom become entangled with the laser field at all? Considering, for sake of argument, that the total decoherence rate can be calculated as Itano claims, can any part of it be attributed to entanglement between the laser field and atom? By implication, if not directly, Itano claims such entanglement is zero. The authors of the criticized work claim it is not, though the entanglement is small [9, 10], agreeing at most that the spontaneous emission calculation gives the correct number for the total decoherence rate, while asserting that its account of the laser-entanglement part of the decoherence is incorrect.

We return to these questions in Sec. VI after presenting our own analysis of the problem of a two-state atom driven by coherent light.

Itano's criticism of Refs. [6, 7] begins with the observation that the Jaynes-Cummings model is inappropriate for treating a free-space atom driven by a quantized field. Potential problems with the model were discussed by Silberfarb and Deutsch [15]. In response it has been pointed out that a careful use of the model can nevertheless yield correct results [9, 10]. We do not intend to focus on the limitations of the Jaynes-Cummings model; we think them amply clear. The fundamental character of the considered physics is that of a scattering process. The Jaynes-Cummings model does not deal with scattering and is inappropriate in this sense, though it can give meaningful results of a perturbative sort. Our plan is to start with a formulation in explicit scattering terms. For this purpose we model the atom and its driving field as cascaded open quantum systems (COQS) [11, 12, 13]. Our model describes the unidirectional cou- pling of the laser output field and a target two-state atom in the Born-Markov approximation. The quantized field interacting with the atom is intrinsically multimode; it supports a propagating laser pulse and an outgoing scattered field. The Lindblad master equation derived by Silberfarb and Deutsch [15] is contained within the model through its forwards scattering terms. While we consider only coherent driving of the atom here, the COQS approach is more general and can treat nonclassical driving fields as well.

\section{CASCADED QUANTUM SYSTEMS}

We begin by briefly reviewing the COQS model. As depicted in Fig. [1 the complete system is comprised of the laser subsystem, denoted $L$, and the target atom subsystem, denoted $A$. The subsystems have free Hamiltonians $H_{L}$ and $H_{A}$, and couple through the quantized electromagnetic field, which is denoted as a reservoir with Hamiltonian $H_{R}$. Free-fields $\hat{\mathcal{E}}_{L}^{\text {in }}$ and $\hat{\mathcal{E}}_{A}^{\text {in' }}$ provide vacuum inputs to subsystems $L$ and $A$, respectively. Subsystems $L$ and $A$ couple unidirectionally through the common channel $\hat{\mathcal{E}}_{L}^{\text {in }} \rightarrow \hat{\mathcal{E}}_{L}^{\text {out }} \rightarrow \hat{\mathcal{E}}_{A}^{\text {in }} \rightarrow \hat{\mathcal{E}}_{F}$. The scattered fields are the forwards scattered field $\hat{\mathcal{E}}_{F}$ and the sideways scattered field $\hat{\mathcal{E}}_{S}$. All fields have units of the square root of photon flux.

A master equation for $L \otimes A$ is derived in the BornMarkov approximation [12]. First, the laser output field in the Heisenberg picture is written as

$$
\hat{\mathcal{E}}_{L}^{\text {out }}(t)=\hat{\mathcal{E}}_{L}^{\mathrm{in}}(t)+\sqrt{2 \kappa_{L}} \hat{a}(t),
$$

where $\hat{a}(t)$ is the annihilation operator for the intracavity mode and $2 \kappa_{L}$ is the cavity linewidth. This field propagates freely to the target atom, which thus couples to the retarded field $\hat{\mathcal{E}}_{L}^{\text {out }}\left(t^{\prime}\right), t^{\prime}=t-l / c$, where $l$ is the distance from $L$ to $A$. Designating the position of the output mirror as $z=0$, the field $\hat{\mathcal{E}}_{L}^{\text {out }}(t)$ is evaluated at $z=0+$. The laser cavity mode couples to the field at $z=0$. Denoting the latter by $\hat{\mathcal{E}}_{L}(t)$, the atom thus couples to the field

$$
\hat{\mathcal{E}}_{L}^{\text {out }}\left(t^{\prime}\right)=\hat{\mathcal{E}}_{L}\left(t^{\prime}\right)+\frac{1}{2} \sqrt{2 \kappa_{L}} \hat{a}\left(t^{\prime}\right)
$$

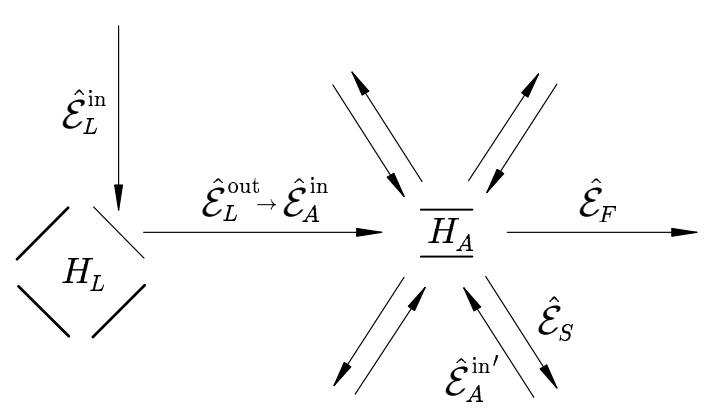

FIG. 1: Schematic diagram of the cascaded system of a two-state atom (Hamiltonian $H_{A}$ ) driven by a coherent laser source (Hamiltonian $H_{L}$ ). The various inputs and outputs are defined in the text. 
We note that the atom and laser cavity mode (at the retarded time) both couple to the reservoir field $\hat{\mathcal{E}}_{L}\left(t^{\prime}\right)$; the atom also couples directly to the intracavity laser field $\frac{1}{2} \sqrt{2 \kappa_{L}} \hat{a}\left(t^{\prime}\right)$ and to the reservoir field $\hat{\mathcal{E}}_{A}^{\text {in' }}$.

Let us now return to the Schrödinger picture and denote the density operator for the entire system, subsystems $L$ and $A$ plus the reservoir $R$, by $\chi(t)$, and define the source-retarded density operator

$$
\chi^{\prime}(t) \equiv U_{L}(l / c) \chi(t) U_{L}^{\dagger}(l / c),
$$

with

$$
U_{L}(l / c) \equiv \exp \left[(i / \hbar)\left(H_{L}+H_{R}+H_{L R}\right)(l / c)\right],
$$

where $H_{L R}$ is the interaction between $L$ and $R$. Then from the reservoir couplings noted above, applied formally at $t^{\prime}=0$, by a standard derivation, the equation of motion for the reduced density operator $\rho^{\prime} \equiv \operatorname{tr}_{R}\left(\chi^{\prime}\right)$ is $11,12,13$

$$
\dot{\rho}^{\prime}=\frac{1}{i \hbar}\left[H_{0}, \rho^{\prime}\right]+\mathcal{L}_{\hat{J}_{F}} \rho^{\prime}+\mathcal{L}_{\hat{J}_{S}} \rho^{\prime},
$$

where $\mathcal{L}_{\hat{O}}$ is the Lindblad superoperator,

$$
\mathcal{L}_{\hat{O}} \equiv \hat{O} \cdot \hat{O}^{\dagger}-\frac{1}{2} \hat{O}^{\dagger} \hat{O} \cdot-\frac{1}{2} \cdot \hat{O}^{\dagger} \hat{O},
$$

and

$$
H_{0}=H_{L}+H_{A}+i \hbar \sqrt{\kappa_{L} \kappa_{A}}\left(\hat{a}^{\dagger} \hat{\sigma}_{-}-\hat{a} \hat{\sigma}_{+}\right),
$$

where $\hat{\sigma}_{+}$and $\hat{\sigma}_{-}$are raising and lowering operators for the atom. The forwards- and side-scattering jump operators are

$$
\hat{J}_{F}=\sqrt{2 \kappa_{L}} \hat{a}+\sqrt{2 \kappa_{A}} \hat{\sigma}_{-}, \quad \hat{J}_{S}=\sqrt{2 \kappa_{A}^{\prime}} \hat{\sigma}_{-} .
$$

The Lindblad $\mathcal{L}_{\hat{J}_{F}}$ enters Eq. (5) through the coupling of $L$ and $A$ to the common (forwards) scattering channel via the field $\mathcal{E}_{L}\left(t^{\prime}\right)$, where a decay rate $2 \kappa_{A}$ is introduced phenomenologically to parameterize the strength of the coupling to the atom; this coupling strength depends on the overlap of the atomic dipole mode with the solid angle subtended by the laser pulse. The Lindblad $\mathcal{L}_{\hat{\mathcal{J}}_{\mathcal{S}}}$ accounts for the interaction of the atom with the additional reservoir field $\hat{\mathcal{E}}_{A}^{\text {in'}}$, and contributes the additional decay rate $2 \kappa_{A}^{\prime}$. The total free-space atomic decay rate is $\gamma=2 \kappa_{A}+2 \kappa_{A}^{\prime}$.

Equations (5)-(8) define the model we adopt as the simplest implementation of the driving of a two-state atom by a quantized field as a scattering process. The model introduces a number of approximations. In particular, the laser field is treated as one-dimensional and single mode, both potentially severe restrictions for a highly focused laser pulse. The model is adequate, however, to address the questions raised, and its generalization is straightforward. Relaxation of the one-dimensional approximation, for example, is discussed in [16].

\section{QUANTUM TRAJECTORIES}

The scattering features of our model are most apparent in a quantum trajectory unraveling of master equation (5) 12, 17]. One imagines performing photoelectric detection of the fields $\hat{\mathcal{E}}_{F}$ and $\hat{\mathcal{E}}_{S}$ and making a record, REC, of the times and type of the detection events. One constructs a conditional evolution, conditioned on the detection record, in which the (unnormalized) stochastic wavefunction $\left|\bar{\psi}_{\text {REC }}\right\rangle$ evolves continuously according to the Schrödinger-like equation

$$
\left|\dot{\bar{\psi}}_{\mathrm{REC}}\right\rangle=\frac{1}{i \hbar} H_{B}\left|\bar{\psi}_{\mathrm{REC}}\right\rangle
$$

with non-Hermitian Hamiltonian

$$
\begin{aligned}
H_{B}= & H_{L}+H_{A}-i \hbar \kappa_{L} \hat{a}^{\dagger} \hat{a}-i \hbar \frac{\gamma}{2} \hat{\sigma}_{+} \hat{\sigma}_{-} \\
& -2 i \hbar \sqrt{\kappa_{L} \kappa_{A}} \hat{a} \hat{\sigma}_{+},
\end{aligned}
$$

and suffers quantum jumps,

$$
\left|\bar{\psi}_{\mathrm{REC}}\right\rangle \rightarrow \hat{J}_{F}\left|\bar{\psi}_{\mathrm{REC}}\right\rangle
$$

$$
\left|\bar{\psi}_{\mathrm{REC}}\right\rangle \rightarrow \hat{J}_{S}\left|\bar{\psi}_{\mathrm{REC}}\right\rangle
$$

determined in a Monte-Carlo fashion with probabilities (per time step $d t$ )

$$
\begin{gathered}
p_{L A}=\left\langle\psi_{\mathrm{REC}}\left|\hat{J}_{F}^{\dagger} \hat{J}_{F}\right| \psi_{\mathrm{REC}}\right\rangle d t, \\
p_{A}=\left\langle\psi_{\mathrm{REC}}\left|\hat{J}_{S}^{\dagger} \hat{J}_{S}\right| \psi_{\mathrm{REC}}\right\rangle d t .
\end{gathered}
$$

Jumps executed by the operators $\hat{J}_{F}$ and $\hat{J}_{S}$ denote the detection of a photon in the forwards- and side-scattered fields, respectively $\left(\hat{\mathcal{E}}_{F}\right.$ and $\hat{\mathcal{E}}_{S}$ in Fig. (1).

In this formulation the unidirectional coupling of the laser source to the target atom is explicit. The last term, proportional to $\hat{a} \hat{\sigma}_{+}$, in Hamiltonian (10) annihilates a laser photon and excites the atom, but there is no term $\hat{a}^{\dagger} \hat{\sigma}_{-}$for the reemission of photons into the laser field. Thus, as Itano pointed out, interaction with the atom does not change the laser field upstream from the atom (the time-retarded field at the source). The atom does, however, absorb photons emitted a retardation time earlier by the laser source. Photons are annihilated downstream from the atom (at the imagined detector) by the jump operator $\hat{J}_{F}$. It is not possible to distinguish between the straight-through laser field and forwards reemission, and this indistinguishability can, in principle, give rise to entanglement. For example, for a Fock state $|n\rangle_{L}$ of the laser mode, under the jump $\hat{J}_{F}$ we have

$$
|n\rangle_{L}|+\rangle_{A} \rightarrow \sqrt{2 \kappa_{L} n}|n-1\rangle_{L}|+\rangle_{A}+\sqrt{2 \kappa_{A}}|n\rangle_{L}|-\rangle_{A},
$$

where $|+\rangle_{A}$ and $|-\rangle_{A}$ denote the upper and lower atomic states. In this regard a laser pulse in a coherent state 
is special, since for a coherent state, as an eigenstate of the annihilation operator $\hat{a}$, there is no entanglement produced by $\hat{J}_{F}$; neither does the continuous evolution (9) produce entanglement, due to the absence of a term $\hat{a}^{\dagger} \hat{\sigma}_{-}$in $H_{B}$. Thus, we see that under coherent excitation the atom does not entangle with the field that drives it (the retarded field at the source), though for excitation by a nonclassical field it generally would. The question of whether there is entanglement with the forwards scattering remains. We return to this question in Sec. VI

We move now to the question of the decoherence rate. The side-scattering quantum jump (11b) shows how decoherence works in quantum trajectory theory for spontaneous emission. The atom jumps to its ground state at a determined rate, which destroys induced coherence. The rate is proportional in our model to $2 \kappa_{A}^{\prime}$, rather than the free-space spontaneous emission rate $\gamma$; the missing $2 \kappa_{A}$ comes from the forwards scattering channel. Decoherence for the forwards scattering operates differently, since the jump operator $\hat{J}_{F}$ does not put the atom in its ground state. In fact, the unique form of the $\hat{J}_{F}$ jump is of interest for a separate reason. In the absence of a term $\hat{a}^{\dagger} \hat{\sigma}_{-}$in the Hamiltonian $H_{B}$, we might wonder how there can be any Rabi oscillation? Rabi oscillations are produced by absorption from and reemission into the driving field; the reemission term is missing.

The answer to this conundrum is that reemission, the deexitation of the atom, is generated, not by any Hamiltonian, but by repeated $\hat{J}_{F}$ jumps 12$]$; it is irreversible, as one might expect for a scattering process. Importantly, for fixed Rabi frequency, the form of the oscillation depends on the ratio $2 \kappa_{A} / \gamma$, i.e., on the degree of focusing of the laser pulse. A weakly (strongly) focused pulse must contain more (fewer) photons to produce the same Rabi frequency. Consequently, the inference about the sate of the atom on detecting a photon in the forwards direction is weaker (stronger), while the frequency of these detections is higher (lower). The inferred conditional evolution of the atomic state then depends on $2 \kappa_{A} / \gamma$ in the manner shown in Fig. 2 A weakly focused pulse containing many photons yields an almost continuous Rabi oscillation of the kind produced by the JaynesCummings Hamiltonian with classical driving field [frame (a)]. Strong focusing and fewer photons yields a ragged oscillation since a stronger inference about the atomic state can be made on the basis of a single photoelectric detection [frame (b)].

It is clear from this discussion that decoherence for a coherently driven two-state atom can be divided into two distinct parts - that due to forwards scattering and that due to scattering out of the forwards channel. The forwards part, moreover, is subject to a $1 / \bar{n}$ effect as the previous authors have claimed [6, 7, 9, 10].

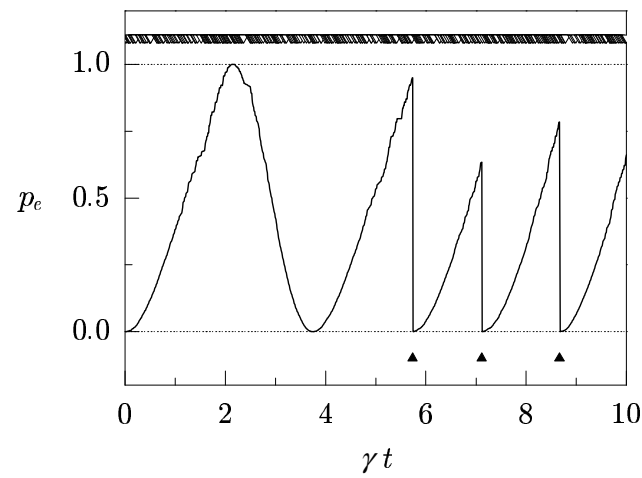

(a)

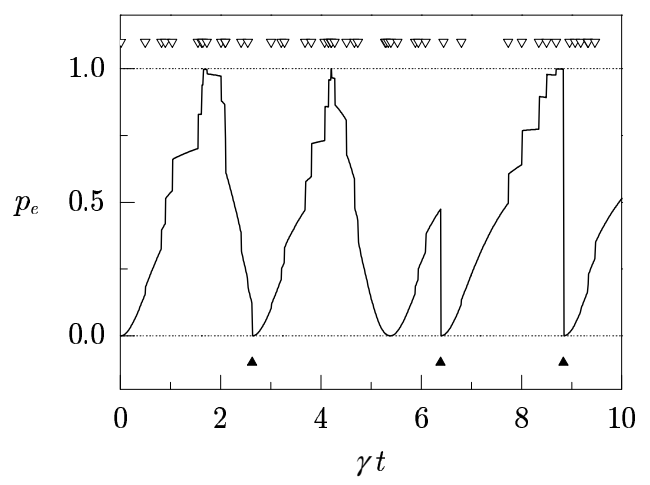

(b)

FIG. 2: Sample Monte-Carlo simulations of the Rabi oscillation of the driven two-state atom in the presence of spontaneous emission, total emission rate $\gamma=2 \kappa_{A}+2 \kappa_{A}^{\prime}$ : for a Rabi frequency $\Omega=\sqrt{\kappa_{L} \kappa_{A}}|\alpha|=2 \gamma$ (for coherent state $|\alpha\rangle_{L}$ ) and forwards scattering rate (a) $2 \kappa_{A} / \gamma=0.04$, (b) $2 \kappa_{A} / \gamma=0.4$. The probability $p_{e}(t)$ to find the atom in the excited state is plotted a function of time. Open (closed) triangles mark the times of forwards- (side-) scattering quantum jumps.

\section{MASTER EQUATION FOR THE ATOM}

Considering the different forms of the quantum jumps in the two channels, it is unclear whether the total decoherence rate may be considered to be due to spontaneous emission alone or not. To resolve this issue we set the quantum trajectory formulation aside and derive a master equation for the atom alone.

We note first, from Eq. (5), that the equation of motion for the reduced density operator of $L, \rho_{L}=\operatorname{tr}_{A}\left(\rho^{\prime}\right)$, is given by the laser master equation

$$
\dot{\rho}_{L}=\frac{1}{i \hbar}\left[H_{L}, \rho_{L}\right]+\mathcal{L}_{\hat{J}_{L}} \rho_{L},
$$

with

$$
\hat{J}_{L}=\sqrt{2 \kappa_{A}} \hat{a}
$$

To generate a coherent state of the intracavity field, we adopt the model Hamiltonian

$$
H_{L}=\hbar \omega_{L} \hat{a}^{\dagger} \hat{a}+i \hbar \kappa_{L}\left[\lambda(t) e^{-i \omega_{L} t} \hat{a}^{\dagger}-\lambda^{*}(t) e^{i \omega_{L} t} \hat{a}\right]
$$

where $\omega_{L}$ is the cavity resonant frequency and $\lambda(t)$ is the complex amplitude of a time-dependent classical current 
driving the cavity mode. In a rotating frame, with frequency $\omega_{L}$, it is readily shown (assuming the initial state to be coherent) that the intracavity field is in the coherent state $|\alpha(t)\rangle_{L}$, with $\alpha(t)$ satisfying the equation

$$
\dot{\alpha}(t)=\kappa_{A} \lambda(t)-\kappa_{A} \alpha(t) .
$$

For an initial vacuum state,

$$
\alpha(t)=\kappa_{A} \int_{0}^{t} d t^{\prime} e^{\kappa_{A}\left(t^{\prime}-t\right)} \lambda\left(t^{\prime}\right) .
$$

We now return to master equation (5). Introducing the density operator in the interaction picture,

$$
\tilde{\rho}(t)=U_{0}^{\dagger}(t) \rho^{\prime}(t) U_{0}(t),
$$

where $U_{0}(t) \equiv \exp \left[-i\left(\omega_{L} \hat{a}^{\dagger} \hat{a}+\omega_{A} \hat{\sigma}_{z} / 2\right) t\right]$, we propose the ansatz

$$
\tilde{\rho}(t)=|\alpha(t)\rangle\langle\alpha(t)| \otimes \rho_{A}(t),
$$

which is suggested by our observation from quantum trajectories that, with the field in a coherent state, the atom does not entangle with its driving field. Substituting the ansatz into Eq. (5), and using Eq. (17) and the relation

$$
\frac{d}{d t}|\alpha(t)\rangle=\left(\dot{\alpha}(t) a^{\dagger}-\frac{1}{2} \frac{d}{d t}|\alpha(t)|^{2}\right)|\alpha(t)\rangle,
$$

we obtain a master equation for the atom,

$$
\dot{\rho}_{A}=\frac{1}{i \hbar}\left[H_{\mathrm{eff}}, \rho_{A}\right]+\mathcal{L}_{\hat{J}_{A}} \rho_{A},
$$

with

$$
H_{\mathrm{eff}}=i \hbar \sqrt{4 \kappa_{L} \kappa_{A}}\left[\alpha^{*}(t) \hat{\sigma}_{-} e^{-i \delta t}-\alpha(t) \hat{\sigma}_{+} e^{i \delta t}\right],
$$

where $\delta \equiv \omega_{A}-\omega_{L}$ and

$$
\hat{J}_{A}=\sqrt{\gamma} \hat{\sigma}_{-} .
$$

Master equation (22), with effective Hamiltonian (23), is a central result. It demonstrates that the atom is effectively driven by a classical field and that the total decoherence rate is determined by the spontaneous emission rate, $\gamma=2 \kappa_{A}+2 \kappa_{A}^{\prime}$, into all $4 \pi$ modes. This is so despite the different appearance of the decoherence for forwards scattering in Fig. 2] and despite the $1 / \bar{n}$ effect in the comparison between frames (a) and (b). We conclude that consideration of the quantum nature of a coherent laser pulse does not uncover any decoherence additional to what is accounted for by spontaneous emission.

\section{DISCUSSION}

Our answer to the first of the two questions raised in Sec. III has just been given: the entanglement reported in [6, 7] does not give rise to decoherence in addition to that due to spontaneous emission in the standard treatment of the driving of an atom by a classical field. The second question from Sec. [II touches on more subtle issues: it asks whether there is entanglement between the atom and the laser field at all, or by extension, is any part of the decoherence attributable to entanglement of the atom and the laser field?

Our comments below Eq. (13) and restated in the factored state (20) give a partial answer to this question. We find no entanglement between the atom and the field that is in direct interaction with the atom - the field at $z=l$ at time $t$ and in the cavity $(z=0)$ at the retarded time $t-l / c$. Such entanglement can arise for certain states of the driving field, but does not arise for a coherent state. There remains the question of entanglement with the field downstream from the atom, the forwardsscattered field. Is there entanglement between the laser pulse and the atom after they interact?

In considering this question, we note first that at the level of the unitarily evolving pure state of the entire system-laser, atom, and scattered field (reservoir)every correlation, no matter how prosaic, is accounted for through entanglement. In the quantum trajectory description, entanglement between the atom and scattered fields is resolved each $d t$-effectively continuously in time as $d t \rightarrow 0$ - and the correlations accounted for through the label REC on the system state. Quantum trajectories are therefore unable to say anything directly about entanglement between the forwards-scattered field and the atom. They do, however, retain the correlations, and hence indicate the presence of entanglement indirectly; and certainly, the atom and scattered fields are correlated, hence entangled at the level of the pure state of the entire system. Even for spontaneous emission (side scattering) the purpose of the quantum jump (11b) is to resolve entanglement at this level while retaining the corresponding correlation (for one kind of measurement on the scattered field) between the scattering record and conditional state. For the forwards-scattered light, correlations are established in a similar way; although in this case they take a very different form, showing, for example, the mentioned $1 / \bar{n}$ effect 12,18$]$. Specifically, the detection of a photon, which projects the atom into its ground state for side scattering, can project the atom into its excited state for forwards scattering [12]. For fixed Rabi frequency, the behavior is controlled -if indirectly - by $1 / \bar{n}$ (more directly, by the degree of focusing of the driving field onto the atom). This difference in the form of the correlations comes from the overlap of the forwards scattering with the incident field, which forms the post-interaction laser pulse. Thus, even without an explicit expression for the state, it is safe to say that at the level of the pure state for the entire system, the atom must be entangled with the post-interaction laser pulse.

As a final comment we should emphasize that the analysis in this paper presumes that the laser source is in a coherent state. The coherent state is imposed by the model Hamiltonian (16), not deduced. It has been sug- 
gested that an operationally coherent laser is not strictly in a coherent state [19, 20, 21, 22, 23, 24]. In principle, the COQS approach can address this issue, by deducing the state of the source from a more realistic laser model. While a deeper analysis along these lines might change what one has to say about the entanglement structure of the pure state of the entire system, the central result that there is no additional atomic decoherence is not expected to change.

This work was supported by the NSF under Grant No. PHY-0099576 and by the Marsden Fund of the RSNZ.
[1] L. M. K. Vandersypen et al., Nature 414, 883 (2003).

[2] S. Gulde et al., Nature 421, 48 (2003).

[3] F. Schmidt-Kaler et al., Nature 422, 408 (2003).

[4] D. Leibfried et al., Nature 422, 412 (2003).

[5] J. I. Cirac and P. Zoller, Phys. Rev. Lett. 74, 4091 (1995).

[6] J. Gea-Banacloche, Phys. Rev. A 65, 022308 (2002).

[7] S. J. van Enk and H. J. Kimble, Quantum Inf. Comput. 2, 1 (2002).

[8] W. M. Itano, Phys. Rev. A 68, 046301 (2003).

[9] J. Gea-Banacloche, Phys. Rev. A 68, 046303 (2003); quant-ph/0212027

[10] S. J. van Enk and H. J. Kimble, Phys. Rev. A 68, 046302 (2003).

[11] M. I. Kolobov and I. V. Sokolov, Opt. Spekstrosk. 62, 112 (1987).

[12] H. J. Carmichael, Phys. Rev. Lett. 70, 2273 (1993).

[13] C. W. Gardiner, Phys. Rev. Lett. 70, 2269 (1993).
[14] B. R. Mollow, Phys. Rev. A 12, 1919 (1975).

[15] A. Silberfarb and I. H. Deutsch, Phys. Rev. A 68, 013817 (2003).

[16] S. J. Enk, Phys. Rev. A 69, 043813 (2004).

[17] H. J. Carmichael, An Open Systems Approach to Quantum Optics, Lecture Notes in Physics, New Series m: Monographs, Vol. m18 (Springer, Berlin, 1993).

[18] P. Kochan and H. J. Carmichael, Phys. Rev. A 50, 1700 (1994).

[19] K. Mølmer, Phys. Rev. A 55, 3195 (1997).

[20] J. Gea-Banacloche, Phys. Rev. A 58, 4244 (1998).

[21] K. Mølmer, Phys. Rev. A 58, 4247 (1998).

[22] T. Rudolf and B. C. Sanders, Phys. Rev. Lett. 87, 077903 (2001).

[23] S. J. van Enk and C. Fuchs, Phys. Rev. Lett. 88, 027902.

[24] B. C. Sanders et al., Phys. Rev. A 68, 042329 (2003). 